\title{
Sub-grid models for heat transfer in gas-particle flows with immersed horizontal cylinders
}

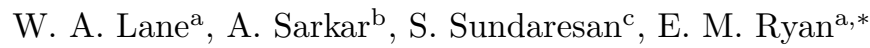 \\ ${ }^{a}$ Boston University, Boston, MA 02215 \\ ${ }^{b}$ Pfizer Inc., Groton, CT 06340 \\ ${ }^{c}$ Princeton University, Princeton, NJ 08540
}

\begin{abstract}
Simulating full-scale heated fluidized bed reactors can provide invaluable insight to the design process. Such simulations are typically computationally intractable due to their complex multi-physics and various lengthscales. While it may be possible to simulate some large-scale systems, they require significant computing resources and do not lend themselves well to design optimization methods. To overcome these problems coarse-grid simulations can be used with supplementary constitutive sub-grid models to approximate the unresolved physics. This study details the development, implementation, and verification of a sub-grid model for heat transfer in gas-particle flows with immersed horizontal cylinders. Using the two-fluid model for multiphase flow, small periodic unit-cell domains were simulated over a wide range of flow and geometry conditions. The results were filtered and fit using nonlinear regression to build a Nusselt correlation based on the solids fraction, solids velocity, cylinder geometry (diameter and spacing), and the Peclet number. The proposed model is highly nonlinear and includes power-law contributions from each parameter. The model was verified using a nearly orthogonal experiment design where the input parameters were varied randomly to generate combinations not previously considered. The predicted filtered Nusselt numbers agreed well with the observed (simulated) values. Work is on-going to further expand the capabilities of the model, including 3D simulations, vertical cylinders, and uncertainty quantification.
\end{abstract}

Keywords: Sub-grid, Filtered, Multiphase, Gas-particle, Heat transfer, CFD

\section{Introduction}

Gas-solid flows are prevalent in many chemical engineering applications, for example, combustion, gasification, fluid-catalytic cracking, and fluid-bed coating/drying. Often these processes are temperature de-

\footnotetext{
*Corresponding author at: Boston University, Department of Mechanical Engineering, 110 Cummington Mall, ENG 416, Boston, MA 02215, USA. Tel.: +1 617353 7767; fax: +1 6173535866.

Email address: ryanem@bu.edu (E. M. Ryan)

Preprint submitted to Chemical Engineering Science

April 30, 2016
} 
pendent, relying on heat transfer cylinders immersed in the flow to regulate the bulk temperature. The multiphase flow and heat transfer occurring within these reactors is extremely complex. The flow behavior is chaotic and the systems never reach true steady states, but rather achieves a statistical steady state characterized by random fluctuations of the flow variables about a mean value. It is often desirable to simulate these systems through computational fluid dynamics (CFD) models as part of the design process. However, in addition to the already complex multiphase physics, these systems span several length scales: the particle diameters are typically hundreds of microns, the heat transfer cylinders are typically a few centimeters, and the reactors are on the order of meters. This combination of multi-physics and multi-scale phenomenon poses a challenging computational problem.

One approach to model such systems is to use high-resolution simulations, with grid cells on the order of a few particle diameters [1]. These simulations are able to explicitly resolve the flow physics and the exact geometry. However, the computational cost scales poorly with device size as prohibitively large numbers of grid cells are needed for bigger geometries.

An efficient solution for modeling these systems is to use low-resolution models coupled with sub-grid constitutive relations to model the unresolved physics. Sub-grid models were originally developed for singlephase turbulence [2]. Turbulent flow properties were studied using highly-resolved but computationallyaffordable periodic domains, and then filtered to construct models for the unresolved turbulent length scales. This approach is now known as large-eddy simulation/methods (LES).

More recently, such sub-grid methods have been extended to simulate gas-particle flows; filtered models have been developed for interphase drag [3-6], cylinder-suspension drag [7, 8], interphase heat transfer [9], and reactive flows [10]. While these filtered-model simulations reduce the ability to predict fine-scale behavior, they can predict bulk flow behavior of large-scale devices with reasonable accuracy and significant computational savings.

The Energy Minimization Multi-Scale (EMMS) method, developed by Jinhai Li [11-13], is an alternative method for approximating the drag in gas-particle flows and has shown great results when implemented with a two-fluid model $[14,15]$. The EMMS method is similar to the sub-grid method in that it uses a constitutive relationship to approximate the flow physics; however, unlike the sub-grid method, it does not depend on a filter or grid size. More recent work has addressed this issue by implementing versions of the EMMS model within a sub-grid framework $[14,16,17]$.

The present research aims to develop filtered constitutive relations to efficiently model the cylinder-suspension heat transfer in a gas-particle fluidized system with immersed cooling/heating tubes. Using this approach, heat-transfer cylinders need not be resolved explicitly in large-scale simulations, but can be replaced with a stationary porous medium and the appropriate sub-grid heat-transfer correlations that mimic the effects of 
the cylinders.

The CFD code and numerical methods used throughout this study are detailed in Section 2. The simulation setup is presented in Section 3 and the filtering methods are presented in Section 4. Sections 5 and 6 discuss the determination of grid and filter sizes, respectively. Model development is derived in Section 7 and verification of the model is addressed in Section 8. Section 9 briefly outlines the implementation of the model for validation and use in CFD codes.

\section{Numerical methods}

This study uses Multiphase Flow with Interphase eXchanges (MFIX), an open source, multiphase, finitevolume CFD package [18]. The system is solved using the Eulerien-Eulerian two-fluid model, where the gas and solid phases are treated as interpenetrating continua. The governing equations for mass, momentum, and energy conservation for the two-fluid model are reviewed in Table 1 and detailed in Section 7 . In lieu of the traditional Kinetic Theory granular energy PDE, Syamlal's simplified algebraic granular energy model [19] and Schaeffer's solids frictional stress model [20] are used. Interphase drag and heat transfer are calculated using the Wen-Yu [21] and Gunn [22] correlations, respectively. The domain was primarily discretized using the second-order Van-Leer scheme; however, the Superbee scheme was used occasionally to overcome convergence issues. Additional models and numerics used in MFIX have been omitted for brevity but are readily available [18]. 
Table 1: Governing equations solved in MFIX [18].

Mass conservation

$$
\begin{aligned}
& \frac{\partial}{\partial t}\left(\phi_{g} \rho_{g}\right)+\nabla \cdot\left(\phi_{g} \rho_{g} \mathbf{v}_{g}\right)=0 \\
& \frac{\partial}{\partial t}\left(\phi_{s} \rho_{s}\right)+\nabla \cdot\left(\phi_{s} \rho_{s} \mathbf{v}_{s}\right)=0
\end{aligned}
$$

Momentum conservation

$$
\begin{aligned}
\frac{\partial}{\partial t}\left(\phi_{g} \rho_{g} \mathbf{v}_{g}\right) & +\nabla \cdot\left(\phi_{g} \rho_{g} \mathbf{v}_{g} \mathbf{v}_{g}\right) \\
& =-\phi_{g} \nabla \cdot \boldsymbol{\sigma}_{g}-I_{g s}+\phi_{g} \rho_{g} \mathbf{g} \\
\frac{\partial}{\partial t}\left(\phi_{s} \rho_{s} \mathbf{v}_{s}\right) & +\nabla \cdot\left(\phi_{s} \rho_{s} \mathbf{v}_{s} \mathbf{v}_{s}\right) \\
& =-\phi_{s} \nabla \cdot \boldsymbol{\sigma}_{g}-\nabla \cdot \boldsymbol{\sigma}_{s}+I_{g s}+\phi_{s} \rho_{s} \mathbf{g}
\end{aligned}
$$

Energy conservation

$$
\begin{aligned}
\frac{\partial}{\partial t}\left(\phi_{g} \rho_{g} C_{p, g} T_{g}\right) & +\nabla \cdot\left(\phi_{g} \rho_{g} C_{p, g} \mathbf{v}_{g} T_{g}\right) \\
& =\nabla \cdot\left(\phi_{g} k_{g} \nabla T_{g}\right)+H_{g s} \\
\frac{\partial}{\partial t}\left(\phi_{s} \rho_{s} C_{p, s} T_{s}\right) & +\nabla \cdot\left(\phi_{s} \rho_{s} C_{p, s} \mathbf{v}_{s} T_{s}\right) \\
& =\nabla \cdot\left(\phi_{s} k_{s} \nabla T_{s}\right)-H_{g s}+\dot{Q}_{s}
\end{aligned}
$$

\section{Simulation setup}

The simulation domain is motivated by a fluidized bed carbon-capture device [23], containing an array of immersed horizontal cylinders (left-hand side of Figure 1). Based on this system, we construct a smaller 2D unit cell sub-domain (right-hand side of Figure 1). This sub-domain is representative of the full system, that is, the periodic unit cell denotes a region in the interior of the bed away from the walls. By using a smaller sub-domain, the full range of possible flow conditions and geometric configurations can be simulated more efficiently. Hereinafter, domain will refer to the reduced 2D periodic unit cell unless explicitly noted otherwise. 


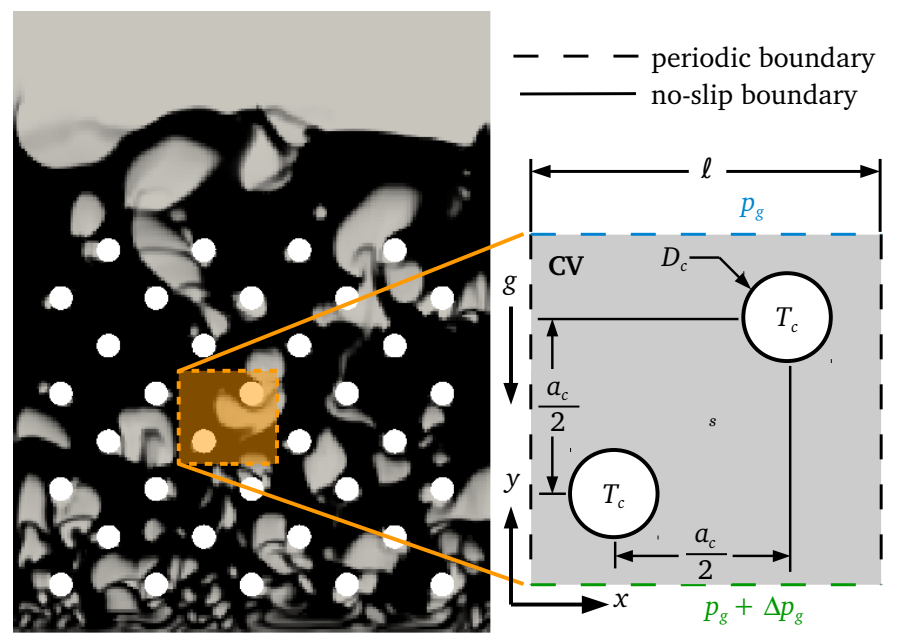

Figure 1: Laboratory-scale fluidized bed example domain (left) and a periodic unit-cell domain (right), where $D_{c}$ is cylinder diameter, $a_{c}$ is cylinder spacing, $T_{c}$ is cylinder surface temperature, $\phi_{s}$ is solids fraction, $p_{g}$ is gas pressure, $\Delta p$ is the gas pressure drop, $g$ is gravitational acceleration, $\ell=a_{c}$ is the length of the domain, and $C V$ is the control volume, shown in gray.

To idealize the flow that occurs in the interior of a fluidized bed, the domain's boundaries are periodic, shown as dashed lines in Figure 1. A macroscopic flow is induced within the domain by imposing a pressure drop $\left(\Delta p_{g}\right)$ along the y-direction, opposite gravity.

By varying the magnitude of the pressure drop we are able to prescribe the various flow velocities typically encountered in a large-scale device. Immersed horizontal heat transfer cylinders are present in the domain. The boundary conditions on the cylinder surfaces are defined as no-slip flow for both gas and solids, and a constant pre-defined surface temperature $T_{c}$ (i.e., Dirichlet boundary condition). The curvature of the immersed cylinders is approximated using MFIX's Cartesian cut-cell feature [24], which truncates cells that intercept curvilinear boundaries, creating quadrilateral and triangular cut-cells, as shown in Figure 2. 


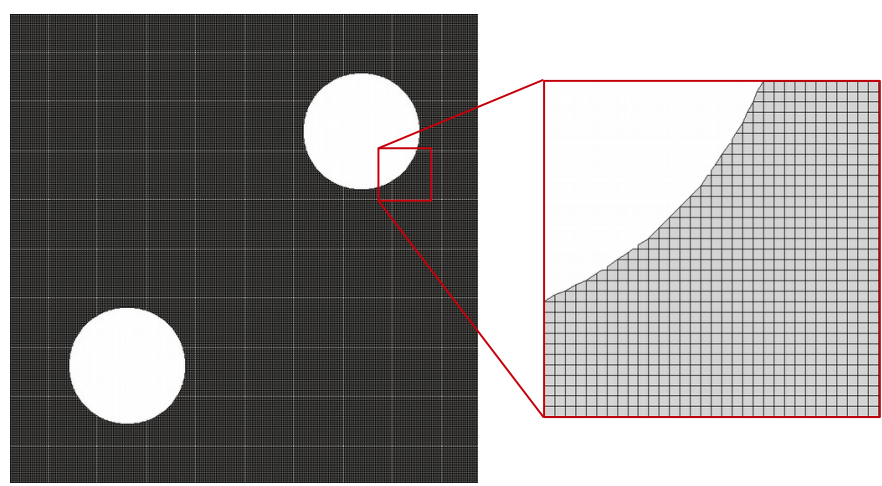

Figure 2: Example of MFIX's cartesian cut-cell method for approximating curved surfaces by truncating nearby cells.

The flow region is initialized as a stationary, homogeneous mixture of gas and solids with an initial suspension temperature of $20^{\circ} \mathrm{C}$ (which is an arbitrary state used in the simulations with no impact on the loss of generality of the results). The gas phase is modeled as air and the solid phase represents carbon-capture solid-sorbent (based on physical properties, Table 2). For the purpose of model derivation and error analysis, a generic source term $\dot{Q}_{s}$ is appended to the solid phase governing energy equation (6) with the form

$$
\dot{Q}_{s}=\phi_{s} \rho_{s} C_{p, s} \dot{\Pi}_{s}
$$

where $\dot{\Pi}_{s}$ is the heat generation rate. The value of the generation rate is arbitrary and does not affect the resulting model. Material properties are adapted from previous studies $[9,23]$ and are listed in Table 2. Additionally we define two characteristic values used to non-dimensionalize quantities: a length and a velocity. These characteristic values are based on the solids terminal velocity $v_{t}$, which has been shown to describe the hydrodynamics of the suspension well in previous gas-particle filtering studies, such as Igci et al. (gas-particle hydrodynamics) [3], Sarkar et al. (gas-particle hydrodynamics with immersed cylinders) [7], Holloway and Sundaresan (reacting gas-particle flows) [10], and Agrawal et al. (gas-particle scalar transport) [9]. The characteristic values are also defined in Table 2. 
Table 2: Material properties and parameters.

\begin{tabular}{|c|c|c|c|}
\hline Parameter & Symbol & Value & Units \\
\hline \multicolumn{4}{|l|}{ Gas phase } \\
\hline Density & $\rho_{g}$ & 1.2 & $\mathrm{~kg} / \mathrm{m}^{3}$ \\
\hline Viscosity & $\mu_{g}$ & $18 \cdot 10^{-6}$ & $\mathrm{~m}^{2} / \mathrm{s}^{2}$ \\
\hline Thermal conductivity & $k_{g}$ & 0.024 & $\mathrm{~W} / \mathrm{m} \cdot \mathrm{K}$ \\
\hline Specific heat capacity & $C_{p, g}$ & 1000 & $\mathrm{~J} / \mathrm{kg} \cdot \mathrm{K}$ \\
\hline \multicolumn{4}{|l|}{ Solid phase } \\
\hline Diameter & $d_{p}$ & $100-300$ & $\mu \mathrm{m}$ \\
\hline Density & $\rho_{s}$ & 441 & $\mathrm{~kg} / \mathrm{m}^{3}$ \\
\hline Thermal conductivity & $k_{s}$ & 0.2 & $\mathrm{~W} / \mathrm{m} \cdot \mathrm{K}$ \\
\hline Specific heat capacity & $C_{p, s}$ & 1000 & $\mathrm{~J} / \mathrm{kg} \cdot \mathrm{K}$ \\
\hline Restitution coefficient & $e$ & 0.9 & - \\
\hline Friction angle & $\theta$ & 28.0 & $\circ$ \\
\hline Generation rate & $\dot{\Pi}_{s}$ & 1 & $\mathrm{~K} / \mathrm{s}$ \\
\hline \multicolumn{4}{|l|}{ Characteristic values } \\
\hline Length & $L^{*}=v_{t}^{2} / g$ & $0.0018-0.146$ & $\mathrm{~m}$ \\
\hline Velocity & $v^{*}=v_{t}$ & $0.133-1.19$ & $\mathrm{~m} / \mathrm{s}$ \\
\hline \multicolumn{4}{|l|}{ Cylinder geometry } \\
\hline Diameter & $D_{c}$ & 3 & $\mathrm{~cm}$ \\
\hline Spacing & $a_{c}$ & $7.5-30$ & $\mathrm{~cm}$ \\
\hline
\end{tabular}

In gas-solid flows, hydrodynamic grid-independent (statistically averaged) results are typically achieved using grid-cell sizes near 10 times the particle diameter $d_{p}[1,4]$. As such, previous work on gas-particle sub-grid model development has employed fine-grid sizes of $16.667 d_{p}[3]$ and $8.333 d_{p}[3,4,7,9]$. However, as will be described in Section 5, accurate resolution of the thermal boundary layer was only achieved with grid-cells no larger than $400 \mu \mathrm{m}$, independent of particle size (eximined in this study). As a result, this study uses a finer grid size of $400 \mu \mathrm{m}$, with the exception of the cut cells which varied and had a minimum cell length of $100 \mu \mathrm{m}$. MFIX employs an adaptive time step algorithm to maintain stability. The time steps were bounded between $10^{-10}$ and $10^{-3}$ seconds, with a mean time step of approximately $10^{-5}$ seconds.

Simulations were run for 10 seconds and sampled at $10 \mathrm{~Hz}$. This provided sufficient data for calculating the thermal equilibrium state. The average runtime of the simulations was approximately 24 hours on 16 processors (Intel Xeon E5-2680 and E5-2650v2 CPUs) but varied with the domain size and specified flow 
conditions.

\section{Filtering methods}

Calculation of the effective cylinder-suspension heat transfer can be accomplished by using an approach similar to that used by Sarkar et al. [7], where the governing equation for net suspension momentum was filtered to calculate the effective cylinder-suspension drag. We use the same top-hat filter and apply it to the governing equation for net suspension energy (the sum of (5) and (6))

$$
\begin{aligned}
\frac{1}{V_{C V}} \int_{C V} \frac{\partial}{\partial t}\left(\phi_{g} \rho_{g} C_{p, g} T_{g}\right. & \left.+\phi_{s} \rho_{s} C_{p, s} T_{s}\right) \mathrm{d} V+\frac{1}{V_{C V}} \int_{C V} \nabla \cdot\left(\phi_{g} \rho_{g} C_{p, g} \mathbf{v}_{g} T_{g}+\phi_{s} \rho_{s} C_{p, s} \mathbf{v}_{s} T_{s}\right) \mathrm{d} V \\
& =\frac{1}{V_{C V}} \int_{C V} \nabla \cdot\left(\phi_{g} k_{g} \nabla T_{g}+\phi_{s} k_{s} \nabla T_{s}\right) \mathrm{d} V+\frac{1}{V_{C V}} \int_{C V} \dot{Q}_{s} \mathrm{~d} V
\end{aligned}
$$

where $V_{C V}$ is volume of the domain's control volume (CV, shown in Figure 1, right). Simplifying the integrals yields

$$
\frac{\partial}{\partial t}\left(\bar{\phi}_{g} \rho_{g} C_{p, g} \widetilde{T}_{g}+\bar{\phi}_{s} \rho_{s} C_{p, s} \widetilde{T}_{s}\right)=\frac{1}{V_{C V}} \oint_{S_{c}}\left(\phi_{g} k_{g} \nabla T_{g}+\phi_{s} k_{s} \nabla T_{s}\right) \cdot \hat{\mathbf{n}}_{c} \mathrm{~d} S+\bar{\phi}_{s} \rho_{s} C_{p, s} \dot{\Pi}_{s},
$$

where $S_{c}$ is the cylinder surface and $\hat{\mathbf{n}}_{c}$ is the unit normal vector to the cylinder surface, pointing inward to the cylinders, overbars denote averages, and over-tildes denote Favre-averages, as detailed in [9]. This simplification is made possible by the divergence theorem; the convective terms can be converted to two surface integrals: one over the periodic boundaries and the other over the cylinders. The surface integrals of the convective terms computed over the periodic boundaries are identically zero. If we analyze the system after it has reached pseudo steady-state the expression is further simplified as the transient term disappears. The remaining surface-integral about the cylinders represents the averaged or filtered volumetric cylinder-to-suspension heat transfer rate, defined as $\bar{Q}_{c s}$, for which we want to constitute a closure equation for

$$
\bar{Q}_{c s}=-\bar{\phi}_{s} \rho_{s} C_{p, s} \dot{\Pi}_{s}
$$

We propose a standard convective heat transfer model for the filtered volumetric cylinder-suspension heat transfer rate 


$$
\bar{Q}_{c s}=\frac{\bar{h}_{c s} A_{c}\left(T_{c}-\widetilde{T}_{\mathrm{susp}}\right)}{V_{C V}}
$$

where $\bar{h}_{c s}$ is the filtered cylinder-suspension heat transfer coefficient, $A_{c}$ is cylinder surface area, $T_{c}$ is the cylinder surface temperature, and $\widetilde{T}_{\text {susp }}$ is the Favre-averaged suspension temperature [9], defined as

$$
\widetilde{T}_{\text {susp }}=\frac{1}{V_{C V}} \int_{C V} \phi_{g} T_{g}+\phi_{s} T_{s} \mathrm{~d} V
$$

It should be noted that the Favre-averaged suspension temperature could also be weighted by density and heat capacity. Such a formulation was considered and when implemented, did not affect the results.

With (10) and (11) we can calculate the filtered cylinder-suspension heat transfer coefficient, $\bar{h}_{c s}$

$$
\bar{h}_{c s}=\frac{\bar{\phi}_{s} \rho_{s} C_{p, s} \dot{\Pi}_{s} V_{C V}}{A_{c}\left(T_{c}-\widetilde{T}_{\text {susp }}\right)}
$$

The cylinder-suspension heat transfer can then be non-dimensionalized by formulating a filtered cylindersuspension Nusselt number,

$$
\overline{\mathrm{Nu}}_{c s}=\frac{\bar{h}_{c s} L^{*}}{k_{s}}
$$

where $L^{*}$ is the characteristic length and $k_{s}$ is the solids thermal conductivity (Table 2).

Using the propagation of error formula [25] we can formulate the standard deviation of the filtered cylindersuspension Nusselt number as

$$
\sigma_{\overline{\mathrm{Nu}}_{c s}}=\sqrt{\left(\frac{\bar{\phi}_{s} \rho_{s} C_{p, s} \dot{\Pi}_{s}}{A_{c}\left(T_{c}-\widetilde{T}_{\mathrm{susp}}\right)^{2}}\right)^{2} \sigma_{\widetilde{T}_{\mathrm{susp}}}^{2}} .
$$

where $\sigma_{\widetilde{T}_{\text {susp }}}$ is the standard deviation of the filtered suspension temperature.

In this work, we seek to construct a correlation for $\overline{\mathrm{Nu}}_{c s}$ for different material properties, flow conditions, and geometry configurations. 


\section{Grid size determination}

A grid sensitivity study was performed to determine the appropriate cell size. When modeling the hydrodynamics of gas-solid flows, typically grid cells are around $10 d_{p}$. However, because thermal boundary layers are typically smaller than the hydrodynamic boundary layers, finer grids are required in order to resolve the heat transfer.

A campaign of 9 simulations was setup to study the convergence of results when varying the grid cell size. Three sizes of particle diameters were considered: 100, 200, and $300 \mu \mathrm{m}$. For each particle size, three grid cell sizes were considered: 800, 400, and $200 \mu \mathrm{m}$. All other material and simulation properties were identical for all simulations (Table 2). The simulations were run for 10 seconds, sufficient time for determining the pseudo steady-state filtered suspension temperature. Snapshots of the solids fraction and solids temperature fields for varying grid sizes are shown in Figure 3.
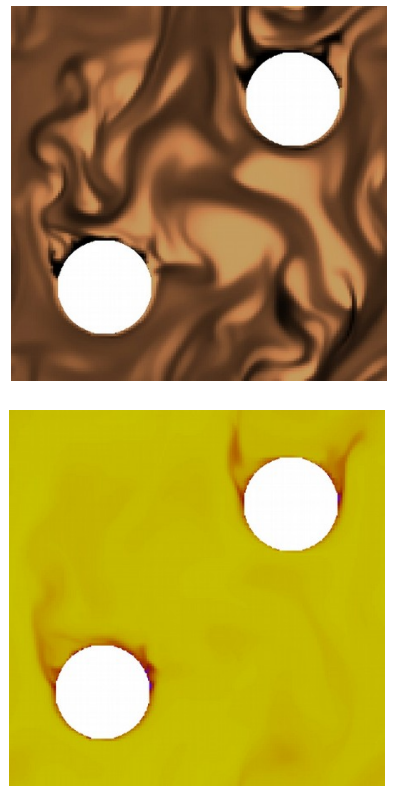

(a) $\Delta_{\text {grid }}=800 \mu \mathrm{m}$
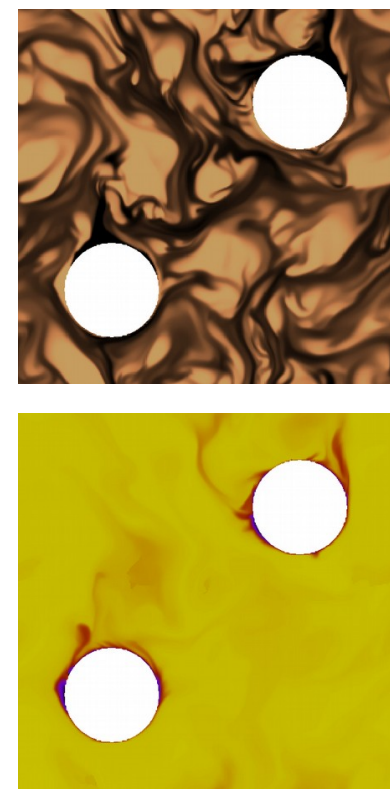

(b) $\Delta_{\text {grid }}=400 \mu \mathrm{m}$
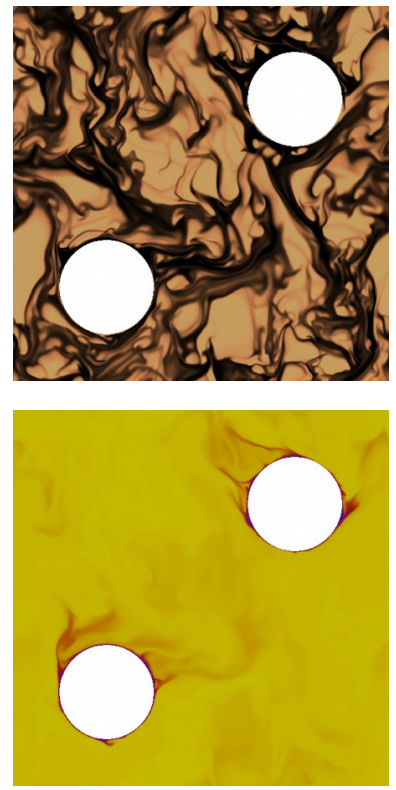

(c) $\Delta_{\text {grid }}=200 \mu \mathrm{m}$
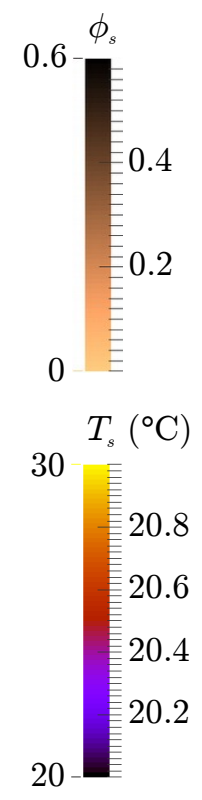

20

Figure 3: Field snapshots of solids fraction (top row) and solids temperature (bottom row) for varying grid sizes with $d_{p}=100 \mu \mathrm{m}, \bar{\phi}_{s}=0.3,\left|\widetilde{\mathbf{v}}_{s}\right|=0.5 \pm 0.02 \mathrm{~m} / \mathrm{s}$, and $a_{c}=12 \mathrm{~cm}$.

Using (14) and (13) the filtered cylinder-suspension Nusselt number was calculated for each simulation and normalized to the $[0,1]$ interval for each particle diameter (Figure 4). 


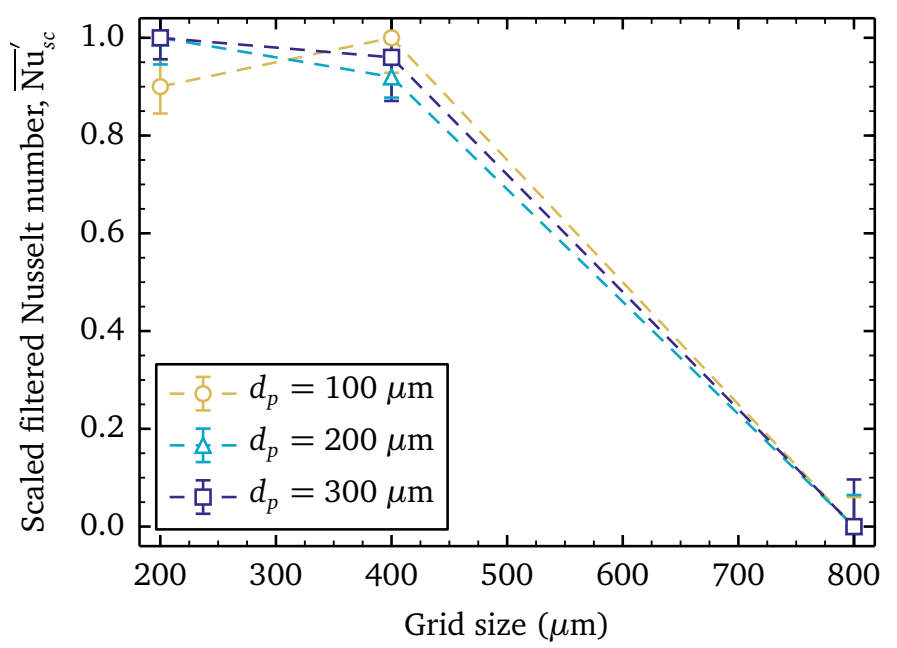

Figure 4: Normalized filtered Nusselt number vs. grid size for particle diameters: 100, 200, and $300 \mu \mathrm{m}$, with $\bar{\phi}_{s}=0.3,\left|\widetilde{\mathbf{v}}_{s}\right|=0.5 \pm 0.02 \mathrm{~m} / \mathrm{s}$, and $a_{c}=12 \mathrm{~cm}$.

From this we see that grid independence is achieved for grid cell sizes around $400 \mu \mathrm{m}$. Unlike the hydrodynamicsonly simulations, for this particular system, the appropriate grid cell size is not a function of particle size. This seems reasonable as the thermal boundary layer is generally a function of the Prandtl number, i.e., related to the thermal properties of the fluid. The remaining simulations in this study were performed using a grid size of $400 \mu \mathrm{m}$.

\section{Filter size determination}

As described in Section 4, we calculate the heat transfer rates by averaging the fields over the defined control volume (also known as the filter). The domain in Figure 1 (right) represents the minimum filter size possible and is referred to as a unit cell. Using this unit cell we can construct domains (filters) of various sizes (Figure 5, left), with the restriction that they are integer multiples of the unit cell.

Filter size is critical for the derivation of the constitutive model. The filter must be large enough to capture the flow and heat transfer characteristics at all length scales. As the filter size increase a more accurate representation of the average physical quantities is expected, until convergence is reached. However, we want to minimize the filter size to reduce overall computation time. Sarkar et al. [7] performed a similar optimization study for the suspension-cylinder hydrodynamics and found a filter size of $2 \ell$ was required; however, they never considered a filter size of $\ell$. To determine the minimum filter size with respect to heat transfer, we consider four different filter sizes: $\ell, 2 \ell, 3 \ell$, and $4 \ell$, where $\ell$ represents the smallest unit-cell size (Figure 5, left). Identical simulation conditions were used for each filter size (Table 2) and 
the simulations were run for 10 seconds, sufficient time for determining the filtered equilibrium suspension temperature.
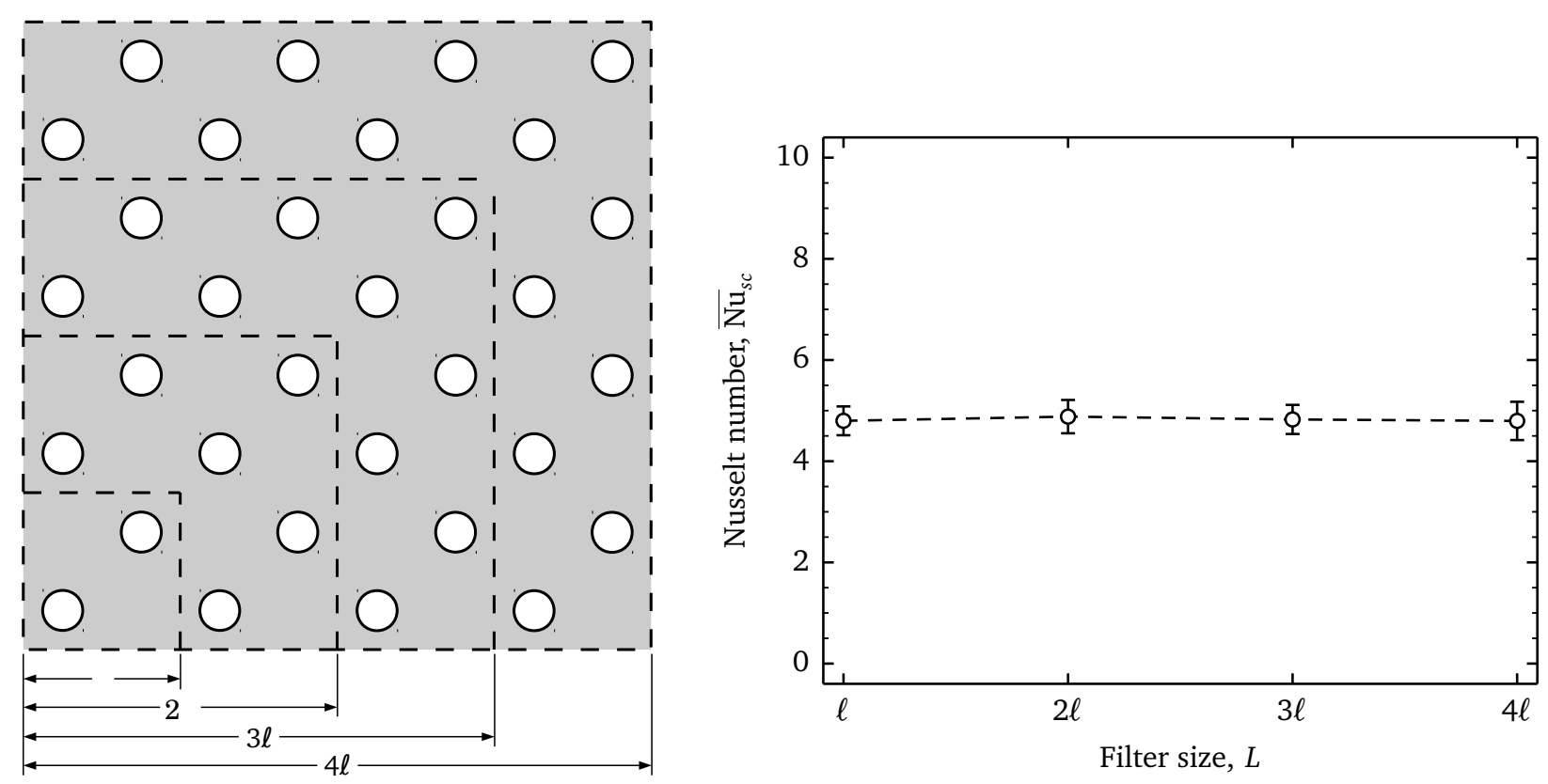

Figure 5: Left: Domains considered to determine the minimum acceptable filter size, expressed as integer multiples of the unit-cell size $\ell$. Right: Nusselt number vs. filter size, with $\bar{\phi}_{s}=0.3,\left|\widetilde{\mathbf{v}}_{s}\right|=0.5 \pm 0.02 \mathrm{~m} / \mathrm{s}$, and $d_{p}=100 \mu \mathrm{m}$.

The simulations were processed using the same method described in the grid study (Section 5). The results showed no significant differences across all filter sizes, indicating that filter size independence is achieved at the smallest filter size, $L=\ell$ (Figure 5). For the remainder of the study, all simulations were run with domains of filter-size $L=\ell=a_{c}$.

\section{Developing the constitutive model}

To develop the sub-grid model, we first complete a dimensional analysis to determine the effective terms for formulation. Using the Buckingham-Pi theorem [26] with the set of variables $\left\{\bar{\phi}_{s},\left|\widetilde{\mathbf{v}}_{s}\right|, \rho_{s}, C_{p, s}, k_{s}, d_{p}, D_{c}, a_{c}, \bar{h}_{s c}\right\}$ and set of basis variables: $\left\{L^{*}, v^{*}, \rho_{s}, k_{s}\right\}$ results in the following dimensionless Pi groups:

$$
\begin{aligned}
& \Pi_{1}=\phi_{s}, \\
& \Pi_{2}=\left|\widetilde{\mathbf{v}}_{s}\right| / v_{t},
\end{aligned}
$$




$$
\begin{aligned}
& \Pi_{3}=D_{c} / a_{c}, \\
& \Pi_{4}=\rho_{s} C_{p, s} v^{*} L^{*} / k_{s}=\overline{\mathrm{Pe}}_{c s}, \\
& \Pi_{5}=\bar{h}_{s c} L^{*} / k_{s}=\overline{\mathrm{Nu}}_{c s},
\end{aligned}
$$

where $\Pi_{2}$ is the dimensionless filtered solids velocity, $\Pi_{3}$ is the ratio of cylinder diameter to spacing, $\Pi_{4}$ is the filtered Peclet number, and $\Pi_{5}$ is the filtered cylinder-suspension Nusselt number. Note that we have chosen to exclude the gas viscosity, and the corresponding dimensionless Prandtl number, from the list above. The cylinder-suspension heat transfer occurs primarily through the particles, and not through the gas and, therefore, the influence of the Prandtl number on $\overline{\mathrm{Nu}}_{c s}$ is not important for particle-laden flows.

According to the Buckingham-Pi theorem, the Nusselt number can be written as a function of the other dimensionless $\Pi$ groups. We choose to correlate $\overline{\mathrm{Nu}}_{c s}$ using a decoupled expression of the form

$$
\overline{\mathrm{Nu}}_{c s}=f_{1}\left(\bar{\phi}_{s}\right) f_{2}\left(\left|\widetilde{\mathbf{v}}_{s}\right| / v^{*}\right) f_{3}\left(D_{c} / a_{c}\right) f_{4}\left(\overline{\mathrm{Pe}}_{c s}\right)
$$

where $f_{1}, f_{2}, f_{3}$, and $f_{4}$ are yet unknown functions of their respective arguments. To determine these functions, we vary the dimensionless groups over a physically relevant range (Table 3) while holding the others at constant values, similar to a factorial experimental design.

Table 3: Pi-group values for simulation campaign.

\begin{tabular}{llll} 
& \multicolumn{3}{c}{ Values } \\
\cline { 2 - 4 } Variable & Nominal & Minimum & Maximum \\
\hline $\bar{\phi}_{s}$ & 0.3 & 0.01 & 0.6 \\
$D_{c} / a_{c}$ & 0.25 & 0.1 & 0.4 \\
$\left|\widetilde{\mathbf{v}}_{s}\right| / v^{*}$ & 3.8 & 0.6 & 7.0 \\
$\overline{\mathrm{P}}_{c s}$ & 531 & 370 & 1,300 \\
\hline
\end{tabular}

First the solids fraction is varied between $0.01-0.6$ to simulate the spectrum from very dilute to very dense systems. The Nusselt number dependence on solids fraction is significant (Figure 6). We see that the behavior is similar to a power law, and we propose the following model form

$$
f_{1}\left(\bar{\phi}_{s}\right) \propto \bar{\phi}_{s}^{\beta_{1}}
$$


where $\beta_{1}$ is an exponent determined through nonlinear regression. Similar behavior is observed with previous models $[3,7,9]$. The model was generated using SciPy's nonlinear regression called curve_fit [27]. The value of $\beta_{1}$ was found to be 0.125 , which agrees well with the data (Figure 6 ). In the limit where the solid fraction goes to zero, a standard single-phase Nusselt number correlation may be used, detailed in Section 9 .

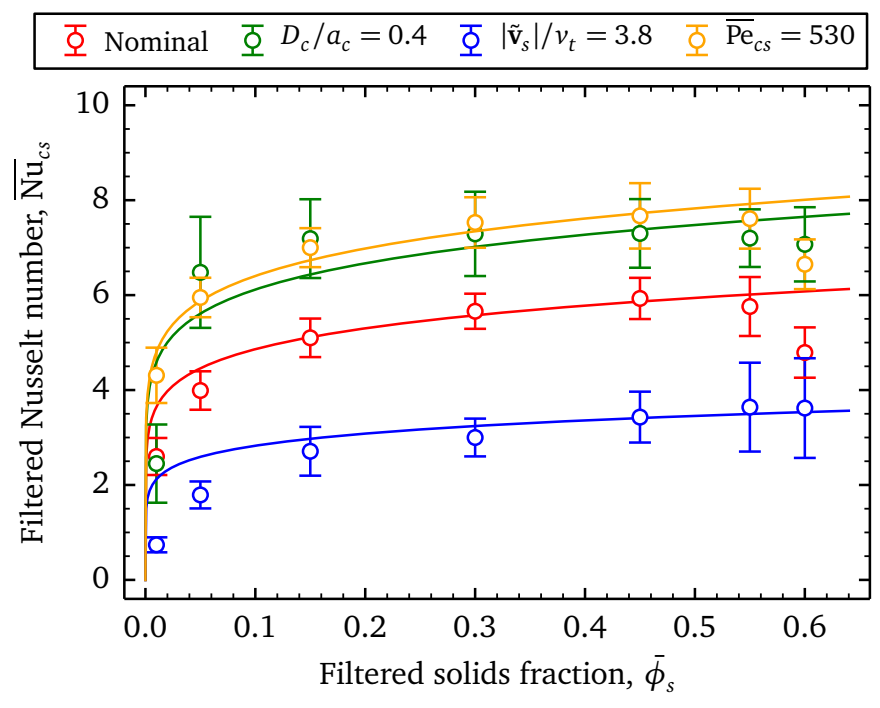

Figure 6: The Nusselt number vs. solids fraction for $D_{c} / a_{c}=0.25,\left|\widetilde{\mathbf{v}}_{s}\right| / v^{*}=3.8$, and $\overline{\mathrm{Pe}}_{c s}=531$, except as noted by the legend. Markers represent simulated data with $\pm \sigma_{\overline{\mathrm{Nu}}_{c s}}$ error bars and the solid lines denote the model fit.

The ratio of cylinder diameter to spacing was varied between $0.1-0.4$, a range often reported in textbooks and literature (see, for example, [28, 29]), by varying the cylinder spacing between $7.5-30 \mathrm{~cm}$ and holding the cylinder diameter at $3 \mathrm{~cm}$. The effect of $D_{c} / a_{c}$ on the Nusselt number was also well-correlated to a power-law and fitted using a polynomial model with an unknown power,

$$
f_{2}\left(\frac{D_{c}}{a_{c}}\right) \propto 1+\beta_{2,1}\left(\frac{D_{c}}{a_{c}}\right)^{\beta_{2,2}}
$$

where $\beta_{2,1}$ and $\beta_{2,2}$ are determined through nonlinear regression. The values of $\beta_{2,1}$ and $\beta_{2,2}$ are found to be 2.94 and 1.76 respectively, which agrees well with the data (Figure 7), capturing the trend and passing through the error bars of the data. Furthermore the proposed form ensures that as the cylinder diameter increases so does the Nusselt number, and inversely as the cylinder spacing increases the term decreases asymptotically. A similar relationship is observed in single phase flow [29]. 


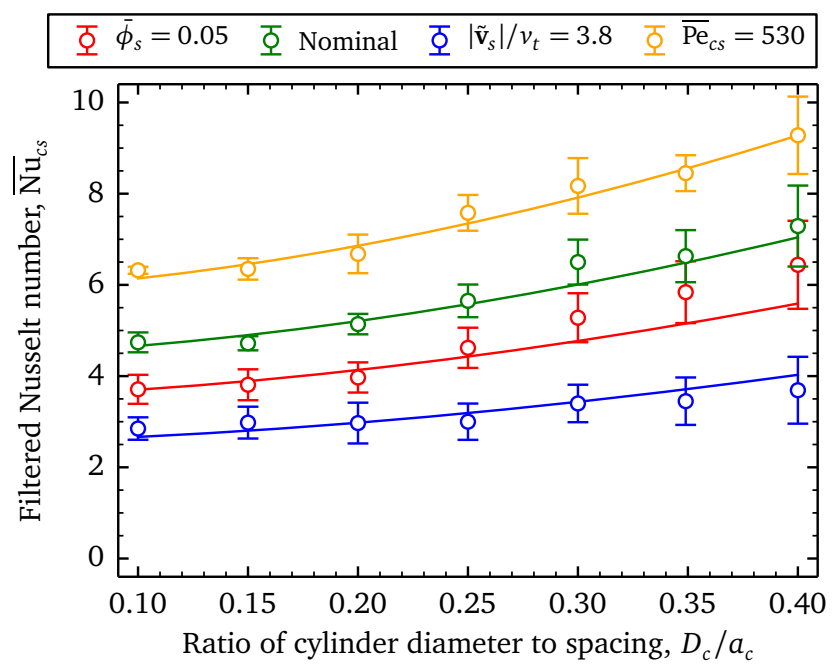

Figure 7: The Nusselt number vs. cylinder diameter-spacing ratio for $\bar{\phi}_{s}=0.3,\left|\widetilde{\mathbf{v}}_{s}\right| / v_{t}=3.8$ and $\overline{\mathrm{Pe}}_{c s}=$ 531 , except as noted by the legend. Markers represent simulated data with $\pm \sigma_{\overline{\mathrm{Nu}}_{c s}}$ error bars and the solid lines denote the model fit.

The dimensionless filtered solids velocity was varied between $0.6-7.0$ by varying the pressure drop to achieve a mean solids velocity of $0.1-1.0 \mathrm{~m} / \mathrm{s}$. We propose a power-law model, analogous to the Reynolds number term present in single-phase flow heat transfer correlations,

$$
f_{3}\left(\frac{\left|\widetilde{\mathbf{v}}_{s}\right|}{v_{t}}\right) \propto\left(\frac{\left|\widetilde{\mathbf{v}}_{s}\right|}{v_{t}}\right)^{\beta_{3}},
$$

where $\beta_{3}$ was found to be 0.341 through nonlinear regression. This form fits the data well and satisfies the limits of going to zero when net flow is zero (Figure 8). 


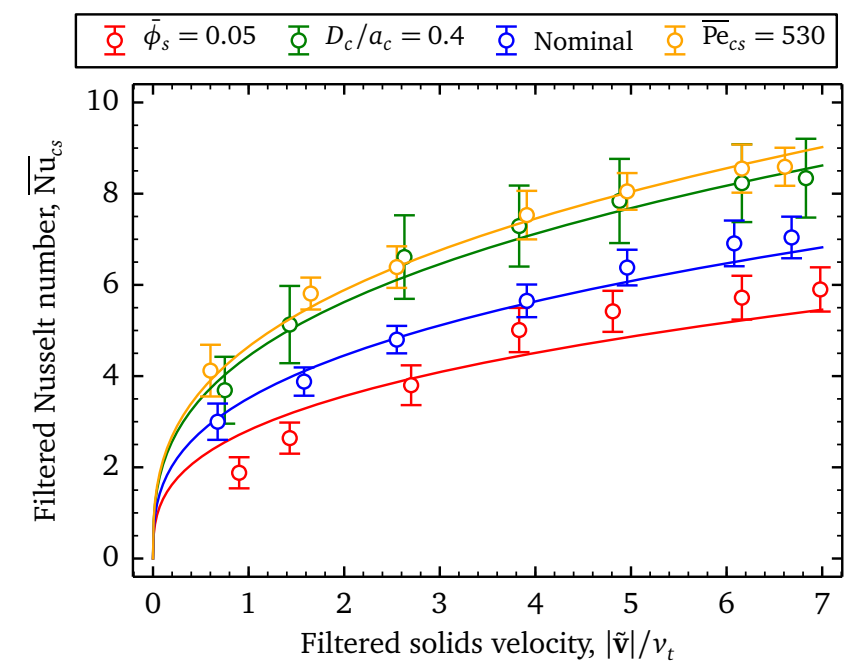

Figure 8: The Nusselt number vs. filtered solids velocity for $\bar{\phi}_{s}=0.3, D_{c} / a_{c}=0.25$, and $\overline{\mathrm{Pe}}_{c s}=531$, except as noted by the legend. Markers represent simulated data with $\pm \sigma_{\overline{\mathrm{Nu}}_{c s}}$ error bars and the solid lines denote the model fit.

The filtered Peclet number was varied between $370-1,330$, by varying the specific heat capacity between $700-2,500 \mathrm{~J} / \mathrm{kg} \cdot \mathrm{K}$. The Nusselt number is fit well using a power law,

$$
f_{4}\left(\overline{\mathrm{Pe}}_{c s}\right) \propto \overline{\mathrm{Pe}}_{c s}^{\beta_{4}},
$$

where $\beta_{4}$ is determined to be 0.353 through nonlinear regression (Figure 9). Thus, we find a relationship between the Nusselt number and thermal properties $\overline{\mathrm{Nu}}_{s} c \propto C_{p} k_{s}^{-1}$, which is similar to the Prandtl number term present in traditional single-phase heat transfer correlations. 


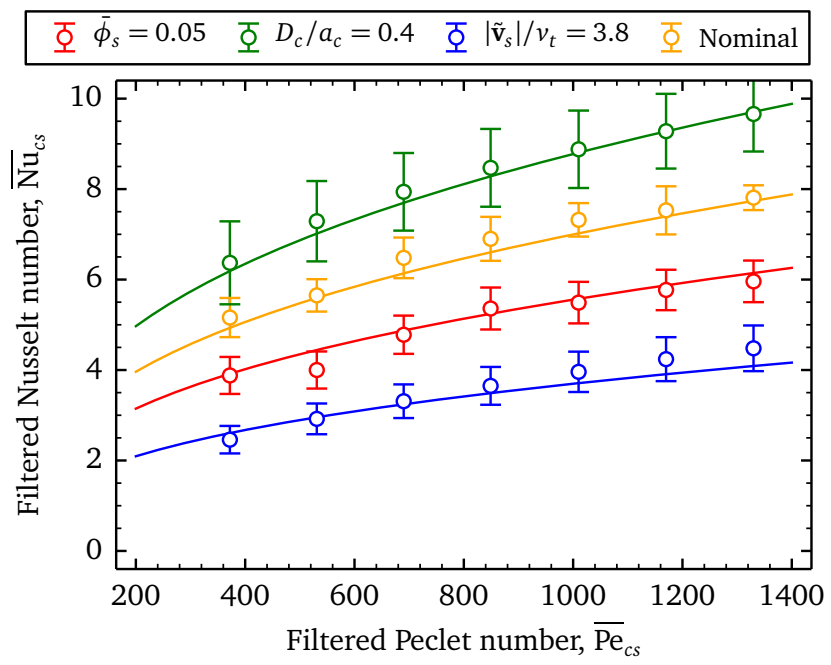

Figure 9: The Nusselt number vs. Peclet number for $\bar{\phi}_{s}=0.3, D_{c} / a_{c}=0.25$, and $\left|\widetilde{\mathbf{v}}_{s}\right| / v^{*}=3.8$, except as noted by the legend. Markers represent simulated data with $\pm \sigma_{\overline{\mathrm{Nu}}_{c s}}$ error bars and the solid lines denote the model fit.

In summary, the individual function forms $f_{1}, f_{2}, f_{3}$, and $f_{4}$, are substituted back in (21) to provide the form of the closure model,

$$
\overline{\mathrm{Nu}}_{c s} \propto \bar{\phi}_{s}^{\beta_{1}}\left[1+\beta_{2,1}\left(\frac{D_{c}}{a_{c}}\right)^{\beta_{2,2}}\right]\left(\frac{\left|\widetilde{\mathbf{v}}_{s}\right|}{v_{t}}\right)^{\beta_{3}} \overline{\mathrm{Pe}}_{c s}^{\beta_{4}} .
$$

This equation is then fit with all of the data simultaneously to determine the final values for all unknowns and the proportionality factor,

$$
\overline{\mathrm{Nu}}_{c s}=0.354 \bar{\phi}_{s}^{0.125}\left[1+2.94\left(\frac{D_{c}}{a_{c}}\right)^{1.76}\right]\left(\frac{\left|\widetilde{\mathbf{v}}_{s}\right|}{v_{t}}\right)^{0.341} \overline{\mathrm{Pe}}_{c s}^{0.353}
$$

\section{Verification}

To ensure the new model performs well under a wide range of mixed conditions, we perform a preliminary verification study using the same periodic unit-cell geometry. Unlike validation which considers the accuracy of an implemented model, this verification solely seeks to examine the accuracy of the algebraic form of the model. Specifically, we wish to evaluate our heat-transfer model for combinations of $\bar{\phi}_{s}, D_{c} / a_{c},\left|\widetilde{\mathbf{v}}_{s}\right| / v_{t}$, and $\overline{\mathrm{Pe}}_{c s}$ values that were not included in the simulation campaign presented in Section 7. To provide thorough 
coverage of the variables' state space, we use nearly orthogonal Latin hypercube (NOLH) sampling to design a verification set of 15 simulations [30,31]. The NOLH values are sampled from the distributions listed in Table 4. The complete list of sampled values for the verification campaign are presented in Table 5.

Table 4: Model verification input parameters distributions.

\begin{tabular}{ll}
\hline Variable & Distribution \\
\hline $\bar{\phi}_{s}$ & $\mathcal{U}(0.01,0.6)$ \\
$D_{c}$ & $\mathcal{U}(0.95,4.5) \mathrm{cm}$ \\
$a_{c}$ & $\mathcal{U}(6,12) \mathrm{cm}$ \\
$d_{p}$ & $\mathcal{U}(50,350) \mu \mathrm{m}$ \\
$C_{p, s}$ & $\mathcal{U}(500,4000) \mathrm{J} / \mathrm{kg} \cdot \mathrm{K}$ \\
\hline
\end{tabular}


Table 5: Model verification input parameters, sampled using nearly orthogonal Latin hypercube [30, 31].

\begin{tabular}{lllll}
\hline$n$ & $\bar{\phi}_{s}$ & $D_{c} / a_{c}$ & $\left|\widetilde{\mathbf{v}}_{s}\right| / v_{t}$ & $\overline{\mathrm{Pe}}_{c s}$ \\
\hline 1 & 0.6 & 0.396 & 2.07 & 4,880 \\
2 & 0.56 & 0.173 & 0.653 & 60,200 \\
3 & 0.53 & 0.4 & 1.85 & 9,850 \\
4 & 0.49 & 0.417 & 0.0798 & $5,370,000$ \\
5 & 0.45 & 0.139 & 0.533 & $3,140,000$ \\
6 & 0.38 & 0.351 & 0.435 & 303,000 \\
7 & 0.34 & 0.237 & 6.38 & 85.7 \\
8 & 0.3 & 0.357 & 0.608 & 155,000 \\
9 & 0.23 & 0.138 & 2.44 & 30,500 \\
10 & 0.19 & 0.171 & 0.353 & 627,000 \\
11 & 0.16 & 0.43 & 3.66 & 582 \\
12 & 0.12 & 0.251 & 21.9 & 16.8 \\
13 & 0.08 & 0.27 & 0.386 & 885,000 \\
14 & 0.05 & 0.33 & 0.93 & 148,000 \\
15 & 0.01 & 0.306 & 0.209 & 530,000 \\
\hline
\end{tabular}

These simulations were run for 10 seconds, and post processed to calculate the observed (i.e., MFIX simulation) and predicted (i.e., model) filtered Nusselt numbers and their uncertainties. At this time there is no uncertainty calculation for the model and as such error is only calculated for the simulation results. Results are shown on a log-log plot (Figure 10) to fit the large range of data. Overall there is good agreement with the predicted values, with only a few outliers but no obvious correlations associated with them. 


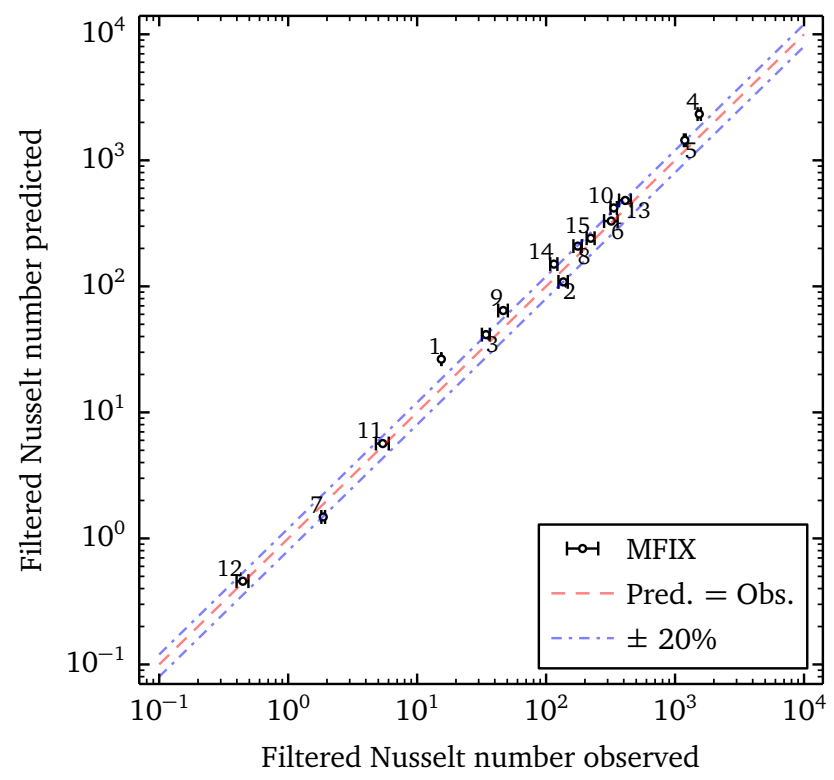

Figure 10: Model verification results showing predicted vs. observed filtered Nusselt numbers. Circles and numbers correspond to the parameter input combinations in Table 5. The light-red dashed line represents an exact match, and the light-blue dashed-dotted lines represent $\pm 20 \%$ bands.

\section{Implementation}

The rate of heat transfer is largely driven by the flow conditions, as such for coarse grid simulations we must ensure that the hydrodynamics are correcly calculated to ensure the accuracy of the heat transfer model. We implement the Igci et al. gas-particle drag model [3] in regions where no cylinders are present and the Sarkar et al. cylinder-suspension drag model [7] in regions containing cylinders. When performing coarse grid simulations the grid cells are often $\mathcal{O}(\mathrm{cm})$, too coarse to allow resolution of the immersed geometry. The cylinder-suspension drag model calculates the drag due to the immersed bodies by implementing a secondary, stationary solid phase, and modifying the governing equations. The stationary solid phase is modeled as a porous media and has a constant phase fraction equal to the volume ratio of the cylinders to the unit cell,

$$
\bar{\Phi}_{c}=\frac{V_{c}}{V_{t o t}}=\frac{\frac{\pi}{4} D_{c}^{2}}{\frac{1}{2} a_{c}^{2}},
$$

where $\bar{\Phi}_{c}$ is the cylinder's filtered phase fraction. With this, we calculate the corrected filtered phasefractions, velocities, and temperatures for the gas and moving solid phase: 


$$
\begin{aligned}
\bar{\Phi}_{i} & =\left(1-\bar{\Phi}_{c}\right) \bar{\phi}_{i}, \\
\widetilde{\mathbf{V}}_{i} & =\widetilde{\mathbf{v}}_{i}, \\
\widetilde{\Theta}_{i} & =\widetilde{T}_{i} .
\end{aligned}
$$

Using (11) and (29)-(31), we can re-write the corrected governing energy equations,

$$
\begin{aligned}
& \frac{\partial}{\partial t}\left(\bar{\Phi}_{g} \rho_{g} C_{p, g} \widetilde{\Theta}_{g}\right)+\nabla \cdot\left(\bar{\Phi}_{g} \rho_{g} C_{p, g} \widetilde{\mathbf{V}}_{g} \widetilde{\Theta}_{g}\right)=\nabla \cdot\left(\bar{\Phi}_{g} k_{g} \nabla \widetilde{\Theta}_{g}\right)+H_{g s}+\bar{Q}_{g c} \\
& \frac{\partial}{\partial t}\left(\bar{\Phi}_{s} \rho_{s} C_{p, s} \widetilde{\Theta}_{s}\right)+\nabla \cdot\left(\bar{\Phi}_{s} \rho_{s} C_{p, s} \widetilde{\mathbf{V}}_{s} \widetilde{\Theta}_{s}\right)=\nabla \cdot\left(\bar{\Phi}_{s} k_{s} \nabla \widetilde{\Theta}_{s}\right)-H_{g s}+\bar{Q}_{c s} .
\end{aligned}
$$

In addition to the newly developed constitutive model for cylinder-suspension heat transfer, we also include a known heat transfer correlation in the gas-phase energy equation [29]. This allows for calculation of heat transfer in the freeboard where no solids may be present. It is implemented similarly to the cylindersuspension model,

$$
\bar{Q}_{g c}=\bar{h}_{g c} A_{c}\left(T_{c}-\bar{T}_{g}\right)
$$

where $\bar{h}_{g c}$ is the filtered gas-cylinder heat transfer coefficient and is calculated from the Nusselt correlation developed by Khan et al. [29]. It is important to note that both of these models operate on the uncorrected variables, $\bar{\phi}_{i}, \widetilde{\mathbf{v}}_{i}$, and $\widetilde{T}_{i}$.

For both source terms the area is calculated by the ratio of unit cell area to grid cell area,

$$
A_{c_{i}}^{\prime}=2 \pi D_{c} \frac{\Delta_{x} \Delta_{y}}{a_{c}^{2}}
$$

where $A_{c_{i}}^{\prime}$ is the area for a single computational cell and $\Delta_{x}$ and $\Delta_{y}$ are cell lengths in the $x$ and $y$ direction. Because the model has been developed, implemented, and tested in $2 \mathrm{D}$, the area calculation is actually $1 \mathrm{D}$ (the circumference of the cylinder). Extensions to 3D are currently under development. 


\section{Conclusion}

Using traditional filtering methods, a sub-grid model for predicting heat transfer in gas-solid flows with immersed horizontal heat transfer cylinders was developed. The new model is analogous to existing singlephase heat transfer correlations but includes the effects of the solids phase (i.e., density, particle diameter, thermal conductivity, specific heat capacity, solids fraction, and velocity). The model was verified with a stochastic test-set of verification simulations that showed good agreement with the observed data.

This research is currently ongoing with efforts towards a more thorough verification, validation, and quantification of uncertainties of the sub-grid models, to be reported in forthcoming publications. Additionally, work has started toward the extension of the model to consider vertical cylinders configurations and 3D simulation domains. However, the simulations required to develop such constitutive relationships are very costly, as such, are left as possible future work.

Additionally, the model is currently being used to simulate a conceptual 1 MWe pilot-scale solid-sorbent carbon-capture reactor [32]; an otherwise computationally intractable system. Preliminary results show great mixing and heat regulation within the system and fair agreement with a previously developed 1D process-level model. This system is undergoing design-optimization to maximize carbon-capture rates based on reaction-kinetics and operating conditions.

\section{Acknowledgements}

This work was funded by the U.S. Department of Energy, Office of Fossil Energy's Carbon Capture Simulation Initiative through the National Energy Technology Laboratory.

\section{Disclaimer}

This presentation was prepared as an account of work sponsored by an agency of the United States Government. Neither the United States Government nor any agency thereof, nor any of their employees, makes any warranty, express or implied, or assumes any legal liability or responsibility for the accuracy, completeness, or usefulness of any information, apparatus, product, or process disclosed, or represents that its use would not infringe privately owned rights. Reference herein to any specific commercial product, process, or service by trade name, trademark, manufacturer, or otherwise does not necessarily constitute or imply its endorsement, recommendation, or favoring by the United States Government or any agency thereof. The views and opinions of authors expressed herein do not necessarily state or reflect those of the United States Government or any agency thereof. 


\section{References}

[1] K. Agrawal, P. N. Loezos, M. Syamlal, S. Sundaresan, The role of meso-scale structures in rapid gassolid flows, Journal of Fluid Mechanics 445 (2001) 151-185.

[2] J. Smagorinsky, General circulation experiments with the primitive equations, Monthly Weather Review 91 (1963) 99.

[3] Y. Igci, A. T. Andrews, S. Sundaresan, S. Pannala, T. O'Brien, Filtered two-fluid models for fluidized gas-particle suspensions, AIChE Journal 54 (2008) 1431-1448.

[4] A. T. Andrews, P. N. Loezos, S. Sundaresan, Coarse-grid simulation of gas-particle flows in vertical risers, Industrial \& Engineering Chemistry Research 44 (2005) 6022-6037.

[5] J. F. Parmentier, O. Simonin, O. Delsart, A functional subgrid drift velocity model for filtered drag prediction in dense fluidized bed, AIChE Journal 58 (2012) 1084-1098.

[6] J. Li, C. Cheng, Z. Zhang, J. Yuan, A. Nemet, F. N. Fett, The EMMS model - its application, development and updated concepts, Chemical Engineering Science 54 (1999) 5409-5425.

[7] A. Sarkar, X. Sun, S. Sundaresan, Sub-grid drag models for horizontal cylinder arrays immersed in gas-particle multiphase flows, Chemical Engineering Science 104 (2013) 399-412.

[8] A. Sarkar, X. Sun, S. Sundaresan, Verification of sub-grid filtered drag models for gas-particle fluidized beds with immersed cylinder arrays, Chemical Engineering Science 114 (2014) 144-154.

[9] K. Agrawal, W. Holloway, C. C. Milioli, F. E. Milioli, S. Sundaresan, Filtered models for scalar transport in gasparticle flows, Chemical Engineering Science 95 (2013) 291-300.

[10] W. Holloway, S. Sundaresan, Filtered models for reacting gasparticle flows, Chemical Engineering Science 82 (2012) $132-143$.

[11] W. Ge, J. Li, Physical mapping of fluidization regimes - the EMMS approach, Chemical Engineering Science 57 (2002) 3993-4004.

[12] J. Li, Multi-scale modeling and method of energy minimization for particle-fluid two-phase flow, 1987.

[13] J. Li, Particle-fluid two-phase flow : the energy-minimization multi-scale method, Metallurgical Industry Press, Beijing, China, 1994.

[14] W. Wang, J. Li, Simulation of gas-solid two-phase flow by a multi-scale cfd approach—of the EMMS model to the sub-grid level, Chemical Engineering Science 62 (2007) 208-231.

[15] N. Yang, W. Wang, W. Ge, J. Li, Cfd simulation of concurrent-up gassolid flow in circulating fluidized beds with structure-dependent drag coefficient, Chemical Engineering Journal 96 (2003) 71-80.

[16] M. Lungu, Y. Zhou, J. Wang, Y. Yang, A CFD study of a bi-disperse gassolid fluidized bed: Effect of the EMMS sub grid drag correction, Powder Technology 280 (2015) 154-172.

[17] J. Wang, Flow structures inside a large-scale turbulent fluidized bed of FCC particles: Eulerian simulation with an EMMS-based sub-grid scale model, Particuology 8 (2010) 176-185.

[18] S. Benyahia, M. Syamlal, T. J. Obrien, Summary of MFIX Equations, from https://mfix.netl.doe.gov/download/mfix/ mfix_current_documentation/MFIXEquations2012-1.pdf, 2012.

[19] M. Syamlal, A review of granular stress constitutive relations, (DOE/MC/21353-2372, DE87006499), Technical Report, National Technical Information Services, 1987.

[20] D. G. Schaeffer, Instability in the evolution equations describing incompressible granular flow, Journal of Differential Equations 66 (1987) 19-50.

[21] C. Wen, Y. Yu, Mechanics of fludization, Chemical Engineering Progress Symposium Series 62 (1966) 100-111.

[22] D. J. Gunn, Transfer of heat or mass to particles in fixed and fluidised beds, International Journal of Heat and Mass Transfer 21 (1978) 467-476.

[23] D. C. Miller, M. Syamlal, D. S. Mebane, C. Storlie, D. Bhattacharyya, N. V. Sahinidis, D. Agarwal, C. Tong, S. E. Zitney, 
A. Sarkar, X. Sun, S. Sundaresan, E. M. Ryan, D. Engel, C. Dale, Carbon capture simulation initiative: A case study in multiscale modeling and new challenges, Annual Review of Chemical and Biomolecular Engineering 5 (2014) 301-323.

[24] J. F. Dietiker, Multiphase Flow with Interface eXchanges Cartesian Grid User Guide, from https://mfix.netl.doe.gov/ download/mfix/mfix_current_documentation/Cartesian_grid_user_guide.pdf, 2015.

[25] H. H. Ku, Notes on the use of propagation of error formulas, Journal of Research of the National Bureau of Standards, Section C: Engineering and Instrumentation 70C (1966) 263-273.

[26] E. Buckingham, On physically similar systems; illustrations of the use of dimensional equations, Physical Review 4 (1914) $345-376$

[27] E. Jones, T. Oliphant, P. Peterson, et al., SciPy: Open source scientific tools for Python, 2001.

[28] F. P. Incropera, D. P. Dewitt, Fundamentals of Heat and Mass Transfer, Wiley, 5th edition, 2002.

[29] W. A. Khan, J. R. Culham, M. M. Yovanovich, Convection heat transfer from tube banks in crossflow: Analytical approach, International Journal of Heat and Mass Transfer 49 (2006) 4831-4838.

[30] S. M. Sanchez, NOLHdesigns spreadsheet. available online via http://harvest.nps.edu/, 2011.

[31] T. M. Cioppa, T. W. Lucas, Efficient nearly orthogonal and space-filling Latin hypercubes, Technometrics 49 (2007) $45-55$.

[32] Z. Xu, C. Lai, P. W. Marcy, J.-F. Dietiker, T. Li, A. Sarkar, X. Sun, Predicting the performance uncertainty of a 1MW pilot-scale carbon capture system after hierarchical laboratory-scale calibration and validation (submitted), Powder Technology (????). 